\title{
MORTALIDADE E PROGNÓSTICO ESPECÍFICO EM PACIENTES COM INSUFICIÊNCIA RENAL AGUDA
}

André luis Balbi*, Daniela Ponce Gabriel, Renata Cristina Barsante, Jacqueline Teixeira Caramori, luis Cuadrado Martin, Pasqual Barreti Trabalho realizado no Departamento de Clínica Médica da Universidade Estadual Paulista - UNESP - Botucatu, SP.

*Correspondência: Depto de Clínica Médica Caixa Postal 584, Rubião Júnior, Botucatu, SP, Cep:18618-970

\begin{abstract}
RESUMO
Vários sistemas de estratificação de risco têm sido estudados com o objetivo de predizer a evolução de pacientes graves com insuficiência renal aguda (IRA).

OBjetivos. Avaliar e comparar as taxas de mortalidade e o escore de severidade individual de necrose tubular aguda (ATNISS) em pacientes que desenvolveram IRA tratados em hospital universitário de atendimento predominantemente terciário. Métodos. Foram estudados prospectivamente 103 pacientes com IRA por necrose tubular aguda (NTA), atendidos no Hospital das Clínicas da Faculdade de Medicina de Botucatu - UNESP, acompanhados até recuperação do quadro renal ou óbito. No momento da primeira avaliação do nefrologista foi aplicado o escore ATN-ISS. Os resultados foram apresentados em média \pm desvio padrão ou mediana, com nível de significância de $5 \%$.

Resultados. Verificamos que $51,4 \%$ eram do sexo masculino, com média de idade de $58 \pm 36$ anos. A mortalidade geral foi de $44,3 \%$, sendo estatisticamente maior nos pacientes provenientes de enfermarias cirúrgicas $(52,7 \%)$ quando comparados àqueles de enfermarias clínicas (35,3\%), e naqueles que realizaram diálise (63,8\%) quando comparados aos de tratamento conservador (23,5\%). 0 escore ATN-ISS apresentou boa confiabilidade, com elevado poder discriminatório (área sob a curva de 0,95) e boa calibração, principalmente a partir do quintil 3 .

ConcLusÃo. Na população estudada, as taxas de mortalidade foram semelhantes àquelas encontradas na literatura. O escore ATN-ISS mostrou ser um índice prognóstico com grande confiabilidade, podendo ser aplicado na prática diária do nefrologista.
\end{abstract}

UNITERMOS: Insuficiência renal aguda. Índice prognóstico. Mortalidade. Fatores de risco. Diálise. Prognóstico.

\section{INTRODUÇÃO}

A mortalidade dos pacientes portadores de insuficiência renal aguda (IRA) permaneceu elevada nas últimas décadas, apesar dos avanços diagnósticos e terapêuticos ocorridos. Mesmo com a utilização de novas técnicas de diálise e recursos nas unidades de terapia intensiva (UTI), o prolongamento da vida do paciente com IRA não significou redução da mortalidade.

Vários sistemas de estratificação de risco têm sido estudados com o objetivo de predizer a evolução de pacientes graves com IRA. Os primeiros trabalhos com o prognóstico da IRA são das décadas anteriores, inicialmente com análise estatística univariada ${ }^{1-3}$ e posteriormente com análise multivariada ${ }^{4-I I}$. Porém, a maioria destes trabalhos foi retrospectiva, sem grupo controle ou com grupo controle pequeno ${ }^{4,6-9}$.

Índices prognósticos, tais como o TISS (Therapeutic Interventions Scoring System) $)^{12}$, SAPS (Simplified Acute Phisiology Score) $)^{13}$, APACHE II e III (Acute Physiology and Chronic Health Evaluation) ${ }^{14}$, MPM (Mortality Prediction Model) $)^{15}$ e PRISM (Pediatric Risk of Mortality) $)^{16,17}$ vêm sendo utilizados, em UTI, para predizer o desfecho de um determinado grupo de pacientes com patologias graves, dentre elas a IRA. D'Ávila et al. ${ }^{18}$ mostraram que o APACHE II, utilizado isoladamente, foi pouco adequado como preditor de IRA em pacientes internados em UTI, pois foi sensível para prever não sobrevivente, mas pouco específico. Fery-Lemonnier-E et al. ${ }^{19}$, relacionand 0 índices prognósticos em IRA, incluindo o APACHE II e SAPS e número de falências de órgãos, concluíram que há em todos eles fontes de ambigüidades nas orientações que devem ser usadas para sua coleta e que a variação entre o valor do índice dado a um paciente por um observador diferia do outro numa magnitude tal que poderia introduzir alterações nos estudos que os utilizassem.

$\mathrm{Na}$ busca de novos índices prognósticos específicos para pacientes que desenvolvem IRA, Liãno et al. ${ }^{20}$ propuseram um escore que pode ser usado também para pacientes internados fora de UTI, denominado escore de severidade individual de necrose tubular aguda (ATN-ISS), obtido imediatamente após a hospitalização ou no momento do atendimento do nefrologista, o que ocorre geralmente até 48 horas após a internação. Este índice foi testado pelo próprio autor em 100 pacientes e, quando comparado com o APACHE II, mostrou excelente confiabilidade. Diversos trabalhos posteriores utilizaram o escore ATN-ISS para pacientes com IRA em situações clínicas diferentes, destacando-se os trabaIhos de Douma et al. ${ }^{5}$, Metha et al. ${ }^{21}$ e, no Brasil, Fernandes et al. ${ }^{22}$, Cendoroglo et al. ${ }^{23} \mathrm{e}$ Teixeira et al. ${ }^{24}$. Nós também utilizamos 0 escore ATN-ISS para randomizar grupos de pacientes com IRA e avaliar métodos de diálise e permanência em UTI, com bons resultados ${ }^{25,26}$. 
Este trabalho tem como objetivos avaliar e comparar as taxas de mortalidade e o escore ATN-ISS em pacientes que desenvolveram IRA tratados em um hospital universitário de atendimento predominantemente terciário.

\section{Métodos}

Foram estudados, prospectivamente, 103 pacientes de ambos os sexos, com idade superior a 12 anos, internados no Hospital das Clínicas da Faculdade de Medicina de Botucatu, UNESP, e acompanhados pelo grupo da IRA da Disciplina de Nefrologia, durante o período de janeiro a dezembro de 2003. Todos possuíam diagnóstico de IRA por necrose tubular aguda (NTA) e estavam distribuídos em leitos de enfermaria geral e em unidades de terapia semi-intensiva e intensiva.

IRA foi definida pela presença de um aumento súbito de creatinina sérica acima de $30 \%$ de seu valor basal, com função renal prévia normal, e NTA caracterizada pela manutenção da insuficiência renal após correção de causas pré-renais e afastadas outras causas parenquimatosas e pós-renais. Quanto à etiologia da IRA, esta pode ser classificada como isquêmica (quando pacientes apresentavam condição hemodinâmica de baixo fluxo renal), nefrotóxica (quando a piora da função renal esteve associada à presença de substâncias nefrotóxicas exógenas ou endógenas) e multifatorial (quando presentes vários insultos renais, dentre eles os quadros sépticos). As indicações de diálise foram aquelas classicamente conhecidas (hipervolemia, uremia, acidose metabólica persistente e hipercalemia importante) e os métodos dialíticos aplicados foram a hemodiálise e a diálise peritoneal.

No momento da avaliação do médico nefrologista foi aplicado o escore ATN-ISS ${ }^{20}$, obtido a partir da equação abaixo e expresso em percentagem. Os pacientes foram acompanhados até resolução do quadro (recuperação da função renal ou óbito).

EscoreATN-ISS = 0,032 (idade em décadas) $-0,086$ (sexo) $-0,109$ (nefrotoxicidade) $+0,109$ (oligúria) $+0,116$ (hipotensão) $+0,122$ (icterícia) $+0,150$ (coma) - 0, I54 (consciência) + 0, 182 (respiração assistida) $+0,21$

Onde:

$\checkmark$ Sexo: I para masculino e 0 para feminino

$\checkmark$ Hipotensão: PA sistólica < $100 \mathrm{mmHg}$ por mais de $10 \mathrm{~h}$, independente do uso de drogas vasoativas

$\checkmark$ Icterícia: se bilirrubina total $>2 \mathrm{mg} / \mathrm{dl}$

$\checkmark$ Foi considerado, para cada item, exceto sexo e idade: $\operatorname{sim}=1$; não $=0$

Os dados foram coletados em protocolo próprio, recebendo prévia autorização do Comitê de Ética em Pesquisa local.

Para análise estatística foi utilizado o teste de Goodman para amostras independentes, com respostas categorizadas, para contraste entre proporções multinormais. Para comparação entre o escore ATN-ISS e a mortalidade em cada subpopulação e população geral foi aplicado o intervalo de confiança. Para avaliação global do escoreATNISS foram utilizadas a discriminação (habilidade do modelo para distinguir os pacientes que sobreviveram daqueles que morreram) e a calibração (capacidade do índice de estimar corretamente a mortalidade ao longo de todos os níveis de risco preditos) ${ }^{27,28}$. A discriminação

\begin{tabular}{|c|c|c|}
\hline \multicolumn{3}{|c|}{ Tabela I - Características da população estudada } \\
\hline & Pacientes (n) & Proporção(\%) \\
\hline Sexomasculino & 53 & 51,5 \\
\hline Médiaidade & \multicolumn{2}{|c|}{$58 \pm 36$ anos } \\
\hline Idade $\leq 60$ anos & 52 & 50,5 \\
\hline Tempodeacompanhamento & \multicolumn{2}{|c|}{$9 \pm 8$ dias } \\
\hline Enfermariacirúrgica & 53 & 51,5 \\
\hline isquêmica & 50 & 51,5 \\
\hline nefrotóxica & 26 & 22,3 \\
\hline multifatorial & 27 & 26,2 \\
\hline Tratamentodialítico & 53 & 51,5 \\
\hline
\end{tabular}

foi obtida pela curva ROC (relação entre sensibilidade e I menos especificidade) e pela área sobre a curva (quanto maior o valor, maior o poder discriminatório do modelo, sendo que valores maiores ou iguais a 0,9 representam excelente discriminação). A calibração foi obtida por meio da análise de curvas de mortalidade prevista e observada com os pacientes agrupados em intervalos de $20 \%$ (quintis). Os dados foram apresentados como média \pm desvio-padrão ou mediana e a significância como $\mathrm{p}<0,05$.

\section{Resultados}

A média de idade dos pacientes estudados foi de $58 \pm 36$ anos ( $50,5 \%$ apresentavam idade menor ou igual a 60 anos), sendo $5 I, 5 \%$ do sexo masculino e $48,5 \%$ feminino. $O$ tempo de acompanhamento pelo nefrologista foi de $9 \pm 8$ dias. Verificamos que $5 \mathrm{I}, 5 \%$ dos pacientes vieram de enfermarias cirúrgicas (incluindo ginecologia, obstetrícia, oftalmologia e otorrinolaringologia) e $48,5 \%$ de enfermarias clínicas. Em relação à etiologia, $51,5 \%$ dos casos foram isquêmicos, $22,3 \%$ nefrotóxicos e 26,2\% multifatoriais. Ainda, 51,5\% dos pacientes realizaram diálise. A Tabela I resume estes resultados.

Amortalidade entre os pacientes estudados foi de $44,3 \%$, sendo de $41,6 \%$ entre o sexo masculino e de $47 \%$ no feminino ( $p>0,05)$. Pacientes maiores de 60 anos apresentaram mortalidade de $51,4 \%$, enquanto naqueles com 60 anos ou menos a mortalidade foi de $37 \%(p>0,05)$. Em relação ao tipo de internação, a mortalidade nas enfermarias cirúrgicas $(52,7 \%)$ foi estatisticamente maior que nas enfermarias clínicas $(35,3 \%)(p<0,05)$. Pacientes com IRA nefrotóxica apresentaram mortalidade $(35,3 \%)$ menor que aqueles com IRA isquêmica $(42,8 \%)$ e multifatorial $(55,7 \%)(p<0,05)$, sendo estas últimas estatisticamente semelhantes. Nos pacientes que realizaram diálise, a mortalidade foi de $63,8 \%$, superior àquela encontrada nos pacientes que realizaram apenas tratamento conservador $(23,5 \%)(p<0,05)$.

A mediana do escore ATN-ISS na população geral foi de 50, I\%. Não houve diferença estatística entre os pacientes do sexo masculino e feminino (mediana de $46,3 \%$ e $54,3 \%$, respectivamente - $p>0,05$ ) e entre aqueles provenientes de enfermarias clínicas (mediana de $52,1 \%$ ) e cirúrgicas (mediana de $43,3 \%$ ) ( $p>0,05)$. Pacientes com idade menor ou igual a 60 anos apresentaram mediana de escore ATN-ISS superior àqueles com idade maior que 60 anos $(37,9 \%$ e $60,1 \%-p<0,05)$. Em relação à etiologia, pacientes com IRA isquêmica 


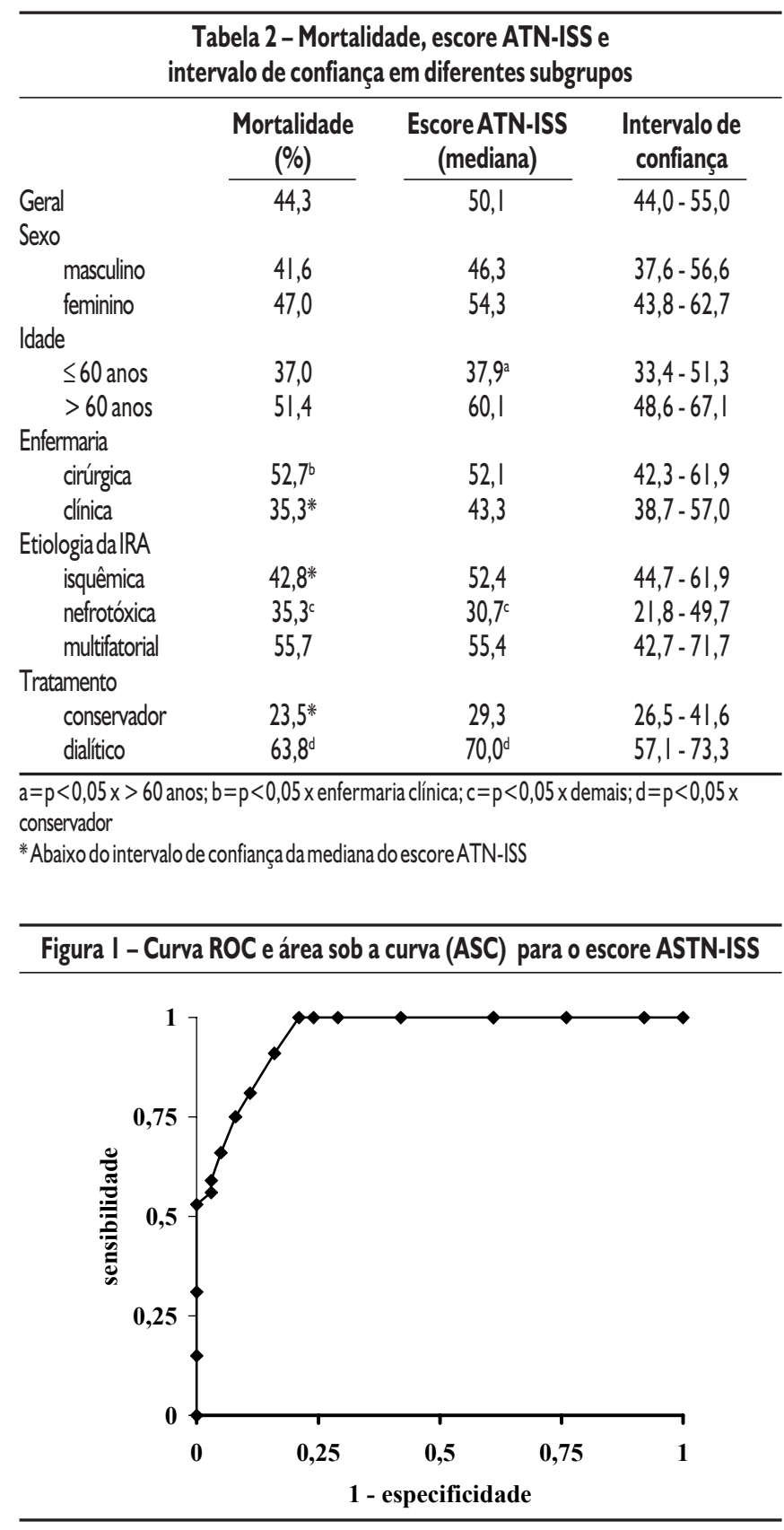

Figura 2-Curvas de mortalidade observada e prevista em cada quintil de risco

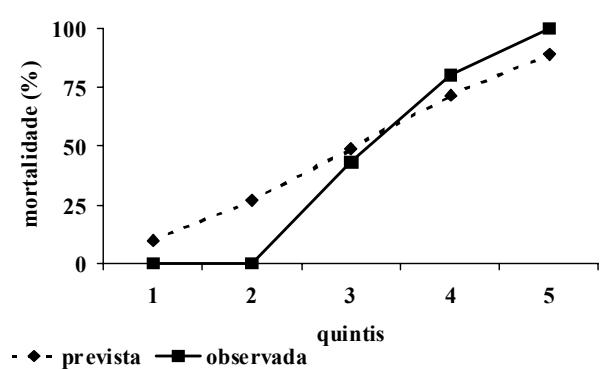

ou multifatorial apresentaram medianas de escore-ATN-ISS semelhantes ( $52,4 \%$ e $55,4 \%$, respectivamente), porém estatisticamente maiores que IRA nefrotóxica (mediana de $30,7 \%)(p<0,05)$. Pacientes que realizaram diálise apresentaram mediana de escoreATN-ISS maior que os que realizaram apenas tratamento conservador $(70 \%$ e $29,3 \%$, respectivamente $-p<0,05$ ).

A Tabela 2 mostra a mortalidade, a mediana do escore ATN-ISSe o intervalo de confiança (limite inferior e superior) do escore ATN-ISS estabelecido para a população estudada e para cada subgrupo. Apenas pacientes provenientes de enfermarias clínicas, com IRA isquêmica e que não realizaram diálise apresentaram mortalidade abaixo dos limites de confiança estabelecidos para o escore ATN-ISS. Não houve subgrupo em que a mortalidade obtida esteve acima dos limites de confiança estabelecidos para o escore ATN-ISS.

Para avaliar a discriminação do escore ATN-ISS foi utilizada a curva ROC, com valor de área sob a curva de 0,95 , conforme demonstrado na Figura I. Para avaliar a calibração do escore ATN-ISS foram comparadas curvas de mortalidade prevista e observada entre os pacientes agrupados em quintis de acordo com o escore ATN-ISS em ordem crescente (quintil $\mathrm{I}=2 \mathrm{I}$ pacientes, $2=20$ pacientes, $3=2 \mathrm{I}$ pacientes, $4=20$ pacientes e $5=21$ pacientes), sendo observado que, a partir do quintil 3, o escore ATN-ISS mostrou excelente calibração. A Figura 2 apresenta as curvas descritas.

\section{Discussão}

Este trabalho mostrou as taxas de mortalidade obtidas em uma população de pacientes com IRA por NTA, comparando-as com 0 escore ATN-ISS em diferentes subgrupos.

Os dados são semelhantes aos obtidos na literatura, principalmente em relação às características da população, com média de idade em torno da quarta e quinta décadas de vida, proporção semelhante entre homens e mulheres e entre pacientes clínicos e cirúrgicos, além do predomínio da etiologia isquêmica sobre as demais.

A mortalidade apresentada esteve dentro do observado na literatura quando se consideram pacientes internados tanto em UTI quanto fora dela, sendo maior nos pacientes que realizaram diálise.

Muitos autores analisaram aspectos demográficos, condições pré-existentes, origem da IRA, débito urinário, valores bioquímicos, complicações e modalidades do tratamento ${ }^{1-3,8-10,29-34}$. Liaño et al..$^{35}$, em 1989, desenvolveram um estudo que utiliza a análise de regressão múltipla de dados clínicos obtidos, pela primeira vez, por nefrologista, sendo capaz de verificar pesos relativos para fatores de risco relevantes para a probabilidade de morte após NTA.

D'Ávila et al. ${ }^{18}$, em nosso meio, estudaram 124 pacientes com IRA internados em UTI, divididos em dois grupos, sobreviventes e não sobreviventes. Após análise de regressão logística multivariada, foram definidos como melhores indicadores de não sobrevida: doença hepática/via biliar, choque, ventilação mecânica, uso de drogas vasoativas, substituição lenta da função renal e o índice APACHE II. Segundo Abdulkader et al ${ }^{36}$, são fatores associados a um pior prognóstico, na evolução da recuperação da função renal, a idade (quanto maior, mais demorada a recuperação da função renal), severidade da doença, presença de lesão renal prévia e realização de sessões de diálise. Lohr 
et al. ${ }^{37}$ mostraram que pressão arterial sistólica menor que $110 \mathrm{mmHg}$, ventilação assistida, insuficiência cardíaca congestiva, sépsis suspeita ou comprovada e disfunção gastrointestinal (hemorragias, obstrução, cirurgia abdominal recente, etc) foram relacionados com a sobrevida. Cantarovich et al. ${ }^{38}$ mostraram, por análise univariada, que na admissão hospitalar de pacientes com IRA, seis fatores de risco para morte foram significativos: idade maior que 55 anos, ventilação mecânica, oligúria, sépsis, choque e aumento de bilirrubinas, enquanto que, por análise multivariada, somente oligúria e sépsis foram fatores de risco significativos. Coritsidis et al. ${ }^{39}$ avaliaram três fatores que poderiam determinar a mortalidade na IRA, comparando-a com o APACHE II (albumina sérica, osmolalidade urinária e sépsis). Para pacientes clínicos houve boa correlação, porém o mesmo não ocorreu para cirúrgicos, sugerindo que sejam populações diferentes.

No presente trabalho, alguns fatores de risco foram identificados como marcadores de maior mortalidade, tais como os pacientes cirúrgicos e realização de diálise, enquanto IRA nefrotóxica esteve associada com menor mortalidade. Foi também avaliado o escore ATN-ISS como índice prognóstico na IRA. Este índice foi descrito por Liaño et al. ${ }^{20}$, mostrando que coma, respiração assistida, hipotensão, oligúria e icterícia tiveram uma influência positiva independente sobre a mortalidade e que etiologia nefrotóxica e consciência normal apresentaram bom prognóstico.

Em nosso trabalho a mediana do escore ATN-ISS foi maior nos pacientes maiores de 60 anos quando comparada com os mais jovens; portadores de IRA isquêmica e multifatorial quando comparados com IRA nefrotóxica; e naqueles que fizeram diálise quando comparados com aqueles em tratamento apenas conservador. Quando foram analisados os intervalos de confiança do escore ATN-ISS e a mortalidade, notou-se que houve semelhança entre mortalidade e índice prognóstico na maioria dos subgrupos estudados, uma vez que somente nos pacientes provenientes de enfermaria clínica, portadores de IRA isquêmica e nos que realizaram tratamento conservador a mortalidade esteve abaixo do intervalo de confiança do escore ATN-ISS. A avaliação global deste escore também mostrou excelente resultado, tanto em relação à curva de discriminação quanto a de calibração. $\mathrm{A}$ área sob a curva de 0,95 mostrou que o escore tem excelente poder discriminatório e a calibração mostrou que este escore é bastante confiável, principalmente a partir do quintil 3 .

Douma et al. ${ }^{5}$, analisando retrospectivamente 238 pacientes adultos em UTI com IRA dialítica, avaliaram sete índices gerais de mortalidade equatro específicos para IRA, concluindo que o APACHE III e o escore ATN-ISS foram aqueles capazes de identificar um grupo de pacientes com quase $100 \%$ de chance de mortalidade, sendo que este último apresentou maior área sob a curva $(0,78)$ e melhor calibração, em todos os quintis analisados. Resultados semelhantes também foram encontrados em estudos brasileiros, tais como aqueles realizados por Fernandes et al. ${ }^{22} \mathrm{e}$ Cendoroglo et al. ${ }^{23}$ que, avaliando - APACHE II e o escore ATN-ISS em pacientes com IRA tratados fora de UTI, mostraram que ambos podem ser usados como índices prognósticos se aplicados no dia da avaliação do nefrologista, porém o escore ATN-ISS foi melhor preditor quando aplicado de modo seriado (admissão do paciente, avaliação do nefrologista e no dia da primeira diálise). Teixeira et al. ${ }^{24}$, estudando 439 casos de IRA, demonstraram que o escore ATN-ISS apresentou boa calibração e discriminação, sendo maiores entre os pacientes que foram a óbito quando comparadas aos que permaneceram vivos. Mais recentemente, porém, Mehta etal. ${ }^{21}$ não obtiveram resultados tão promissores com o escoreATN-ISS ao estudar, de modo prospectivo, 605 pacientes com diagnóstico de IRA em UTI. Neste trabalho há apresentação de novo índice desenvolvido pelo próprio grupo, que mostrou melhores resultados.

\section{Conclusão}

Aimportância destes vários índices prognósticos pode ser demonstrada em muitos aspectos, tais como comparar diferentes serviços, a evolução de pacientes semelhantes submetidos a tratamentos diversos, o controle de qualidade interno das atividades em diferentes períodos e a mortalidade esperada e observada. Nosso trabalho mostrou uma boa associação entre o escore ATN-ISS e a mortalidade nos pacientes acompanhados, o que nos permite concluir que trata-se de índice com grande confiabilidade, podendo ser aplicado na prática diária do nefrologista, não descartando, porém, a necessidade de desenvolvimento de índice prognóstico próprio do serviço.

Conflito de interesse: não há.

\section{SUMMARY}

AssesSMENT OF MORTALITY AND SPECIFIC INDEX IN ACUTE RENAL FAILURE

A variety of systems of risk factor stratification have been studied to predict the outcome in acute renal failure (ARF).

OBJECTIVES. Assess and compare mortality and the Acute Tubular Necrosis Individual Severity Score (ATN-ISS) in patients with AFR treated in a university hospital.

METHODS. A prospective analysis was made of 103 patients with a diagnosis of intrinsic ARF admitted to the Hospital das Clinicas da Faculdade de Medicina de Botucatu, UNESP. Patients were followed up until recovery of renal function or death. The Score ATN-ISS was recorded during the first hours of the assessment by a nephrologist. Results were reported as median or mean $\pm S D$, with statistical significance of $p<0.05$.

RESULTS. Fifty-one percent of patients were male with a mean age of 58 \pm 36 years. Forty-four percent died in the hospital. Mortality was higher in patients from the surgical wards (52.7\%) and in patients who were treated with dialysis (63.8\%). The score ATN-ISS showed a good confidence level, with high discriminatory power (area under the curve of 0.95) and good accuracy.

Conclusions. Mortality in this study was comparable to that found in literature. The ATN-ISS was shown to be a prognostic index with a high confidence level that could be routinely applied by nephrologists to patients with AFR. [Rev Assoc Med Bras 2005; 5 I (6): 318-22]

KEY wORDS: Acute renal failure. Prognostic index. Mortality. Risk factors. Dialysis.

\section{REFERÊNCIAS}

I. Hall JW, Johnson WJ, Maher FT, Hunt JC. Immediate and long-term prognosis in acute renal failure. Ann Intern Med I 970;73:5 I 5-2 I.

2. Kennedy AC, Burton JA, Luke RG, Bruggs JD, Lindsay RM, Allison MEM, et al. Factors affecting the prognosis in acute renal failure: a survey of $25 \mathrm{I}$ cases. J Med I 973;42:73-86. 
BaLBI AL ET AL.

3. Stott RB, Ogg CS, Cameron JS, Bewck M. Why the persistently high mortality in acute renal failure? Lancet 1972;2:75-9.

4. Cioffi WG, Ashikaga T, Gamelli RL. Probability of surviving postoperative acute renal failure. Development of a prognostic index. Ann Surg 1984;200:205-II.

5. Douma CE, Redekop WK, Van der Meulen JHP, Olden RWV, Haeck J, Struijk DG, et al. Predicting mortality in intensive care patients with acute renal failure treated with dialysis. J Am Soc Nephrol 1997;8: I II-7.

6. Bullock ML, Armen AJ, Finkelstein M, Keane WF. The assessment of risk factors in 462 patients with acute renal failure. Am J Kidney Dis 1985;5:97-103.

7. Lien J, Chan V. Risk factors influencing survival in acute renal failure treated by hemodialysis. Arch Intern Med I 985; 1 45:2067-69.

8. Corwin HL, Teplick RS, Schreiber MJ, Fang LS, Bonventre JV, Coggins $\mathrm{CH}$. Prediction of outcome in acute renal failure. Am J Nephrol 1987;7:8-12.

9. Lange HW, Aeppli DM, Brown DC. Survival of patients with acute renal failure requiring dialysis after open heart surgery: early prognostic indicators. Am Heart J I 987; I I 3: I I 38-43.

10. Montoliu J, Campistol JM, Cases A, Lens XM, Revert L. Mortalidad y factores prognósticos de supervivencia en la insuficiencia renal aguda grave que requiera dialysis. Nefrologia 1989;9: I52-8.

II. Rasmussen HH, Pitt EA, Ibels LS, Meneil DR. Prediction of outcome in acute renal failure by discriminant analysis of clinical variables. Arch Intern Med 1 985; | 45:2015-8.

12. Knaus WA, Draper EA, Wagner DP, Zimmerman JE. APACHE II: A severity of diseases classification system. Crit Care Med 1985; 13:818-29.

13. Le GallJR, Loirat P, Alperovitch A, Glaser P, Granthil C, Mathieu D, et al. A simplified acute physiology score foi ICT patients. Crit Care Med 1984; 12:975-7.

14. Keene AR, Cullen DJ. Therapeutic Intervention Scoring System: uptade 1983. Crit Care Med 1983; I I: I-3.

I5. LemeshowS, Teres D, Pastides H, Avrunin JS, Steingrub JS. A method for predicting survival and mortality of ICU patients using objectively derived weights. Crit Care Med 1985; 1 3:5 I 9-25.

16. Pollack MM, Ruttimann UE, Getson PR. The Pediatric Risk of Mortality (PRISM) Score. Crit Care Med I 988; 16: I I I 0-6.

17. Sneff M, Knaus WA: Predicting patient outcome from intensive care: a guide to APACHE, MPM, SAPS, PRISM, and other prognostic systems. J Intensive Care Med 1990;5:33-52.

18. D'Ávila DO, Traezel M, Glock L. Insuficiência renal aguda tratada em unidade de tratamento intensivo (análise de 124 pacientes consecutivos). JBras Nefrol I997; | 9:2 I-31.

19. Fery-Lemonnier-E, Landais-P, Loirat-P, Kleinknecht-D, Brivet-F. Evaluation of severity scoring systems in ICUs-translation, conversion and definition ambiguities as a source of inter-observer variability in Apache II, SAPS and OSF. Intensive Care Med 1 995;21:356-60.

20. Liãno F, Gallego A, Pascual J, García-Martín F, Teruel JL, Marcén R, et al. Prognosis of acute tubular necrosis: an extended prospectively contrasted study. Nephron 1993;63:21-3.

21. Mehta RL, Pascual MT, Gruta CG, Zhuang S, Chertow GM. Refining predictive models in critically ill patients with acute renal failure. J Am Soc Nephrol 2002; 13: I 350-7.
22. Fernandes N, Cendoroglo M, Batista PBP, Balda C, Schor N, Stella SR. Uso do escore prognóstico APACHE II para pacientes com IRA tratados fora da Unidade de Terapia Intensiva (não UTI). J Bras Nefrol 2000;22:75.

23. Cendoroglo M, Roque A, Batista PB, Santos OFP, Stella SR, Draibe AS. Serial prognostic score indexes in Acute Renal Failure (ARF): best performance of scores obtained at the time of referral to the nephrologist. J Bras Nefrol 2000;22:6.

24. Teixeira A, Roque A, Fernandes N, Balda C, Stella SR. Insuficiência renal aguda tratada em unidade de terapia intensiva: epidemiologia e fatores prognósticos. J Bras Nefrol 2000;22:75.

25. Balbi AL, Muraro CB, Barsante RC, Kocchi AC, Barretti P. Insuficiência renal aguda por necrose tubular aguda: efeitos do método dialítico sobre a mortalidade J Bras Nefrol 200 I;23(2 supl 4):4 I.

26. Balbi AL, Muraro CB, Barsante RC, Kocchi AC, Barretti P. Insuficiência renal aguda dialítica por necrose tubular Aguda: a permanência em UTI reduz a mortalidade? J Bras Nefrol 200 I;23(2 supl 4):42.

27. LameshowS, Lê GallJR. Modelling the severity of illness: a system uptade. JAMA 1994; 272: 1049-55.

28. HanleyJA, McNeil BJ. The meaning and use of the area under the receiver operating characteristic (ROC) curve. Radiology I 982; 143:29-36.

29. Abreo K, Moorthy V, Osborne M. Changing patterns and outcome of acute renal failure requering hemodialysis. Arch Intern Med 1986; |46:|338-4|.

30. Hou HS, Bushinsky DA, Wish JB, Cohen JJ, Harring JT. Hospital-acquired renal insufficiency: a prospective study. Am J Med I 983;74:243-8.

31. Wilkins RG, Faragher EB. Acute renal failure in an intensive care unit. Incidence, prediction and outcome. Anaesthesia 1983;8:628-34.

32. Rasmussen $\mathrm{HH}$, Ibels LS. Acute renal failure: multivariate analysis of causes and risk factors. Am J Med I 982;73:2 I I-8.

33. Knaus WA, Draper EA, Wagner DP, Zimmerman JE. Prognosis in acute organ-system failure. Ann Surg 1 985;202:685-93.

34. Shen PF, Zhang SC. Acute renal failure and multiple organ system failure. Arch Surg 1987; | 22: I |31-3

35. Liaño F, García-Martin F, Gallego A, Orte L, Teruel JL, Marcén R, et al. Easy and early prognosis in acute tubular necrosis: a forward analysis of 228 cases. Nephron 1989;5 I:307-I 3.

36. Abdulkader RCRM, Costa MC. Recuperação funcional pós necrose tubular aguda. J Bras Nefrol 1996; 18: I 75-9.

37. LohrJW, McFairlaine MJ, Grantham JJ. A clinical index to predict survival in acute renal failure patients requering dialysis. Am J Kidney Dis 1988; I I:254-9.

38. Cantarovich F, Verho MT. A simple prognostic index for patients with acute renal failure requering dialysis. Renal Failure 1996; 18:585-92.

39. Coritsidis GN, Guru K, Ward L, Bashir R, Feinfeld DA, Carvounis CP. Prediction of acute renal failure by "bedside formula" in medical and surgical intensive care patients. Renal Failure 2000;22:235-44.

Artigo recebido: 13/07/04 Aceito para publicação: 17/09/04 